\title{
Correction to: The role of hybridisation in the origin and evolutionary persistence of vertebrate parthenogens: a case study of Darevskia lizards
}

\author{
Susana N. Freitas ${ }^{1,2,6} \cdot$ D. James Harris ${ }^{2}$ Neftalí Sillero ${ }^{3}$ - Marine Arakelyan ${ }^{4} \cdot$ Roger K. Butlin $^{1,5}$. \\ Miguel A. Carretero ${ }^{2}$
}

Published online: 23 September 2019

(C) The Author(s), under exclusive licence to The Genetics Society 2019

\section{Correction to: Heredity}

https://doi.org/10.1038/s41437-019-0256-5
Following the publication of this article the authors noted an incorrect version of Fig. 5 had been included. The correct version has now been added to the HTML and PDF versions of the article.

These authors contributed equally: Roger K. Butlin, Miguel A. Carretero

The original article can be found online at https://doi.org/10.1038/ s41437-019-0256-5

Susana N. Freitas

freitas.sn@gmail.com

1 Department of Animal and Plant Sciences, The University of Sheffield, Sheffield S10 2TN, UK

2 CIBIO Research Centre in Biodiversity and Genetic Resources, InBIO, Universidade do Porto, Campus de Vairão, Rua Padre Armando Quintas, No. 7. 4485-661 Vairão, Vila do Conde, Portugal

3 CICGE Centro de Investigação em Ciências Geo-Espaciais, Faculdade de Ciências da Universidade do Porto, Observatório Astronómico Prof. Manuel de Barros, Alameda do Monte da Virgem, 4430-146 Vila Nova de Gaia, Portugal

4 Yerevan State University, Alek Manoogian, 1, 0025 Yerevan, Armenia

5 Department of Marine Sciences, University of Gothenburg, 40530 Gothenburg, Sweden

6 Present address: Department of Ecology and Evolution, University of Lausanne, Lausanne, Switzerland 

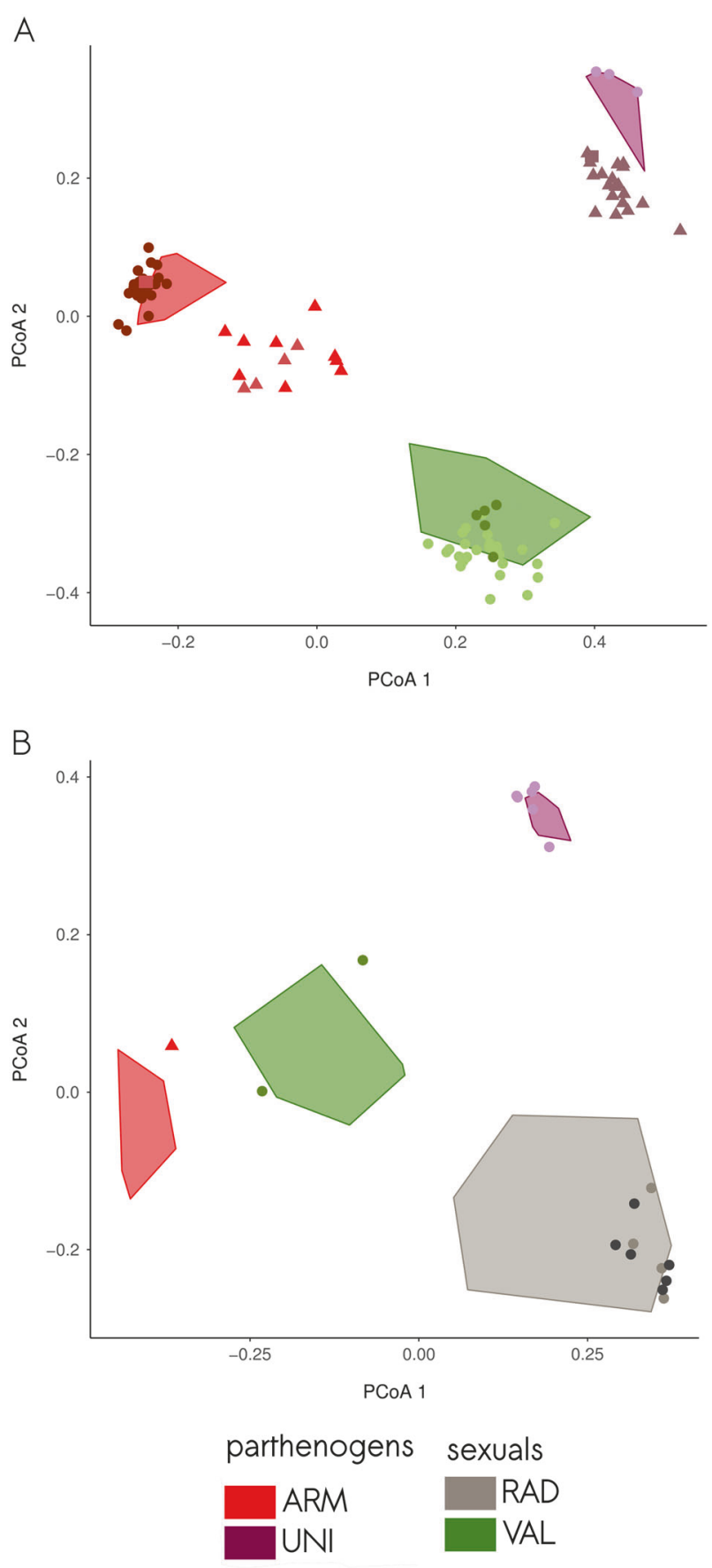

Fig. 5 